\title{
In-vitro and in-vivo regulation of Candida albicans morphogenesis and pathogenesis by probiotic bacterium - Pediococcus acidilactici.
}

\section{Abstract NO: P103}

Zahra Zare, kamiar Zomorodian, Sasan Rezaei,Marjan Motamedi,Davoud Mehrabani, Kazem Ahmadikia Department of Parasitology and Mycology, School of Medicine, Tehran University of Medical Sciences, Tehran, Iran Center of basic research in infectious diseases, Department of Parasitology and Mycology, School of Medicine, Shiraz University of Medical Sciences, Shiraz, Iran

\section{Objectives: Candida species is known as opportunistic yeast} that could cause candidiasis in susceptible indivituals. Oral candidiasis is one of most frequent form of this infection that caused by Candida species in particular, Candida albicans which normally reside on human mucosal surfaces. The transition of C.albicans, from budding yeast to filamentous hyphae allows for covalent attachment to oral epithelial cells, followed by biofilm formation, invasion and tissue damage. It has been suggested that biofilm formation by C.albicans play important role in its pathogenesis. Hence, the effect of Pediococcus acidilactici on the growth, biofilm formation and expression of genes related to morphogenesis and pathogenesis of $C$.albicans were investigated in vitro as well as in animal model.

Methods: Inhibitory activity of Pediococcus acidilactici on Candida species was evaluated by broth microdilution method based on CLSI protocol. Moreover, inhibition of C.albicans biofilm formation by P.acidilactici was measured using XTT reduction assay. Also, expression of adhesion-related gene (ALS1), hyphal cell wall protein gene critical to biofilm formation (HWP1), secreted aspartyl proteinase(SAP6), morphogenesis pathway regulatory gene (EFG1) were analyzed by RT-PCR in the treated yeast cells with different concentrations of P.acidilactici. The experimental activity of the probiotic bacterium in the prevention or treatment of oral candidiasis was also assessed in an animal model by both culture and histopathological methods

Results: $P$. acidilactici inhibited the growth of different species of candida at concentration ranging from 8 to $256 \mu \mathrm{g} / \mathrm{mL}$. Furthermore, this probiotic bacterium inhibited the formation of $C$. albicans biofilm in a dose dependent manner.

Table 1: inhibitory effect of $P$. acidilactici on biofilm formation.

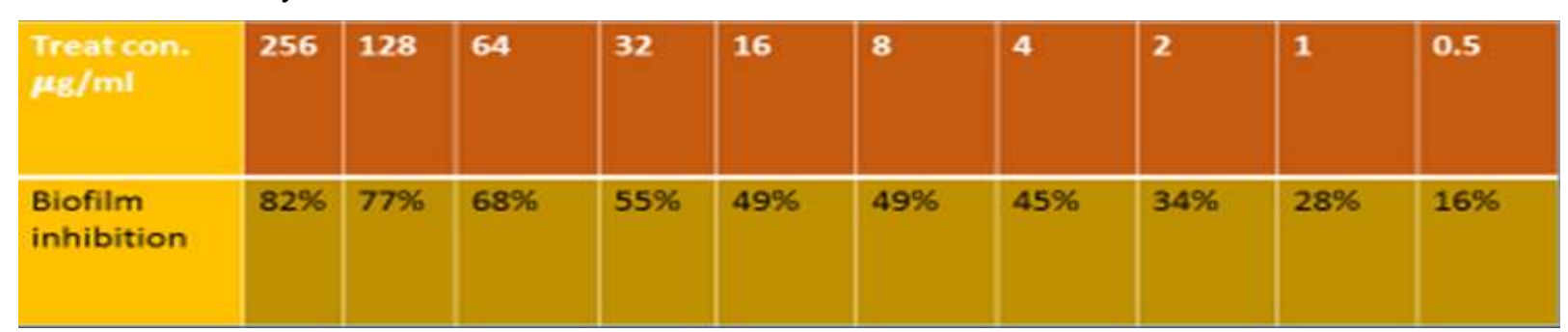

Significant reductions in adherent cells were observed. RT-PCR analysis of $C$.albicans yeast treated with different concentration of probiotic bacterium showed reduction of ALS1, SAP6, EFG1 and HWP1 genes(below chart).

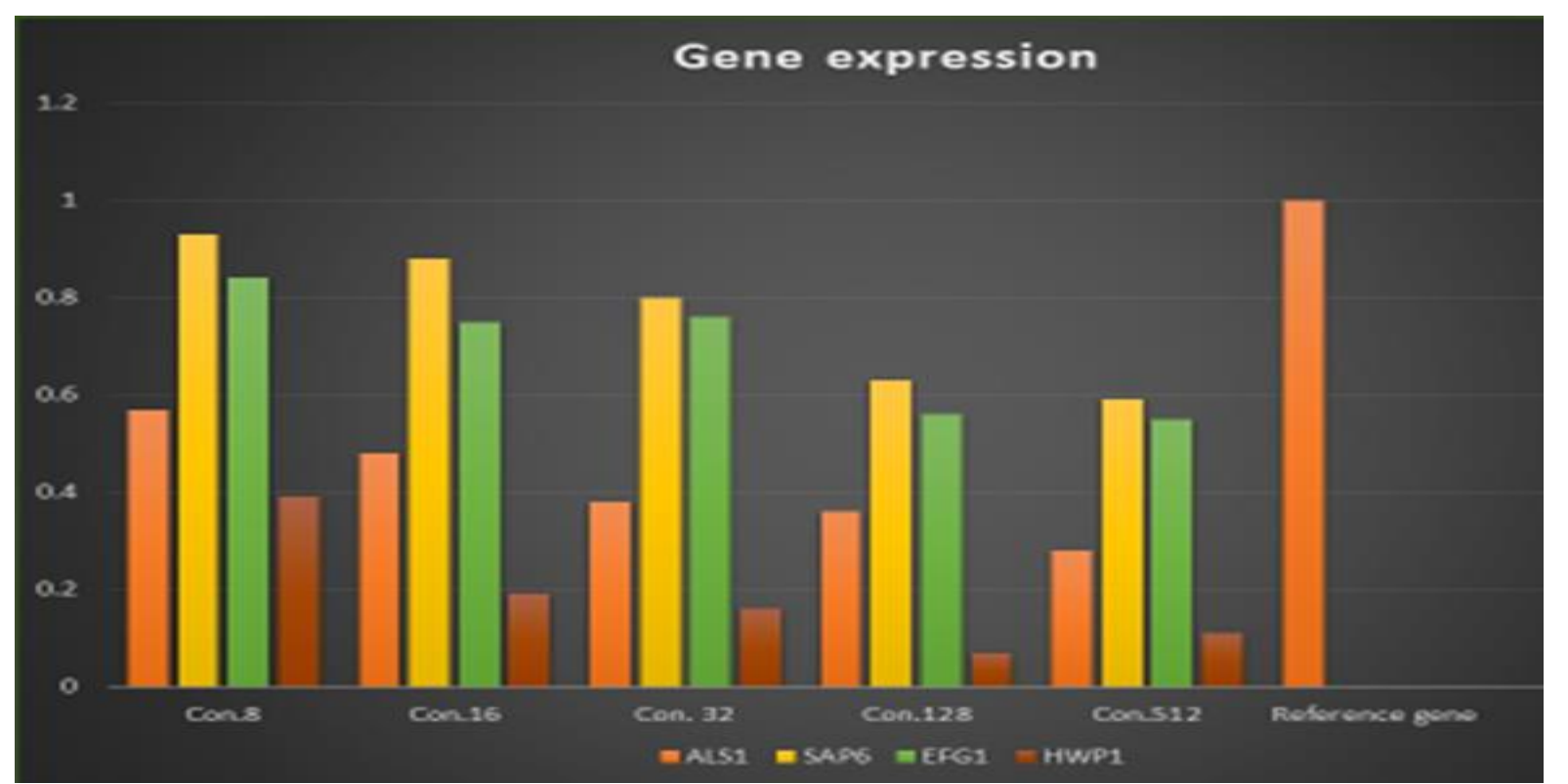

The $P$. acidilactici significantly decreased the CFUs in mice receiving this probiotic treatment compared to those of control group. oral administration of $15 \mathrm{mg} / \mathrm{ml}$ and $30 \mathrm{mg} / \mathrm{ml}$ of $P$.acidilactici respectively resulted in an obviously significant difference from the water -treated control group. The tongues of mice administered $P$. acidilactici showed fewer lesions than the tongues of the control mice . in the mice that were administered $P$.acidilactici at $30 \mathrm{mg} / \mathrm{ml}$, there was a significant decrease in fungal burden compared to that for the untreated control. Histopathological analyses showed that Candida colonization declined in the mice following administration of probiotic in a therapeutic trial. Fewer PAS-staining mycelial elements were found to invade the oral epithelium of tongues treated with $P$.acidilactici than the tongues in the control group(FIG1,2). FIG1

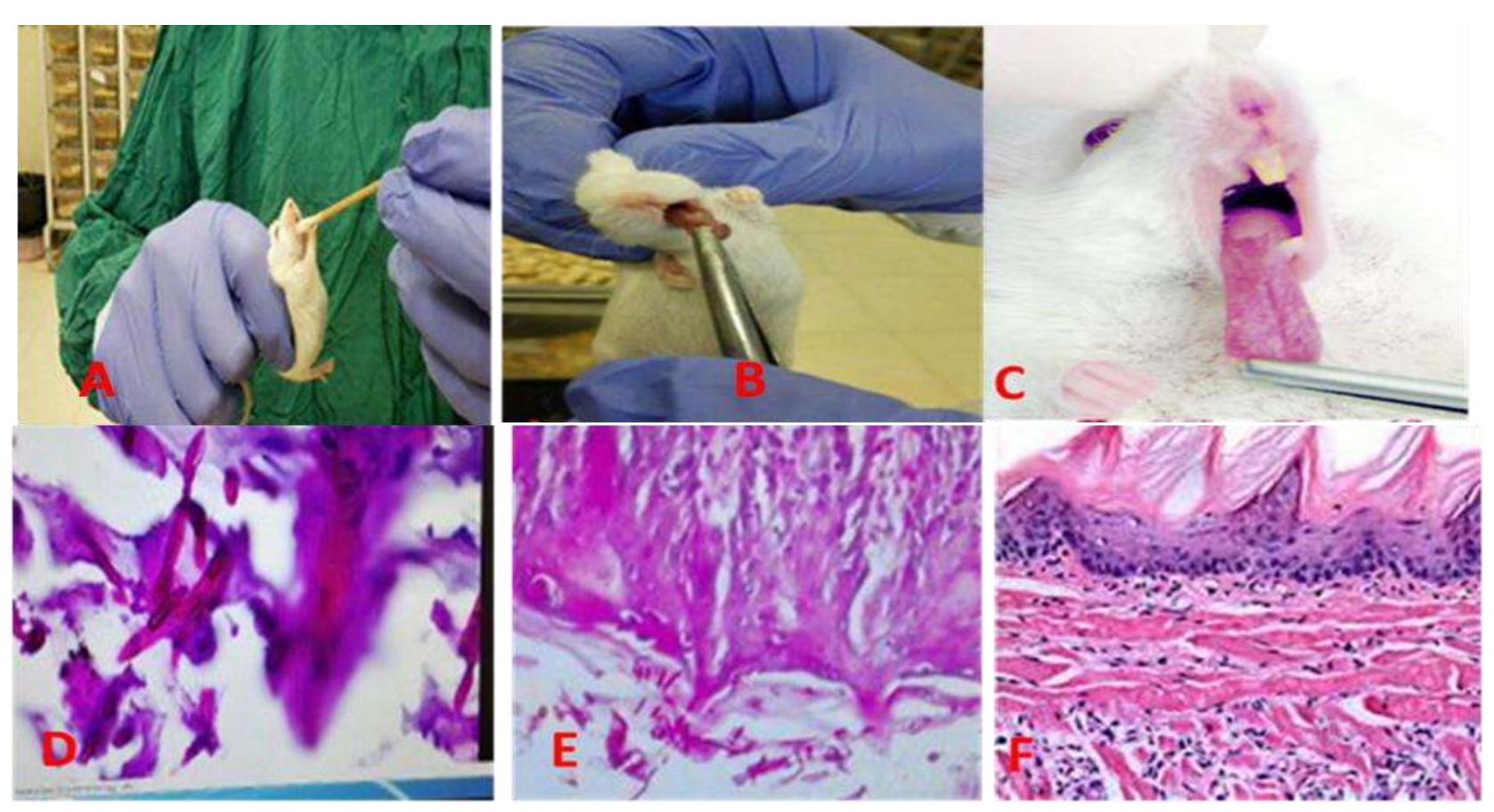

$(A, B, C)$ Initial preparation of laboratory animals. Histology sections of mouse tongues inoculated with C. albicans (D) given $7.5 \mathrm{mg} / \mathrm{ml} P$. acidilactici, (E) control mouse, (F) given $30 \mathrm{mg} / \mathrm{ml} P$. acidilactici .

FIG2

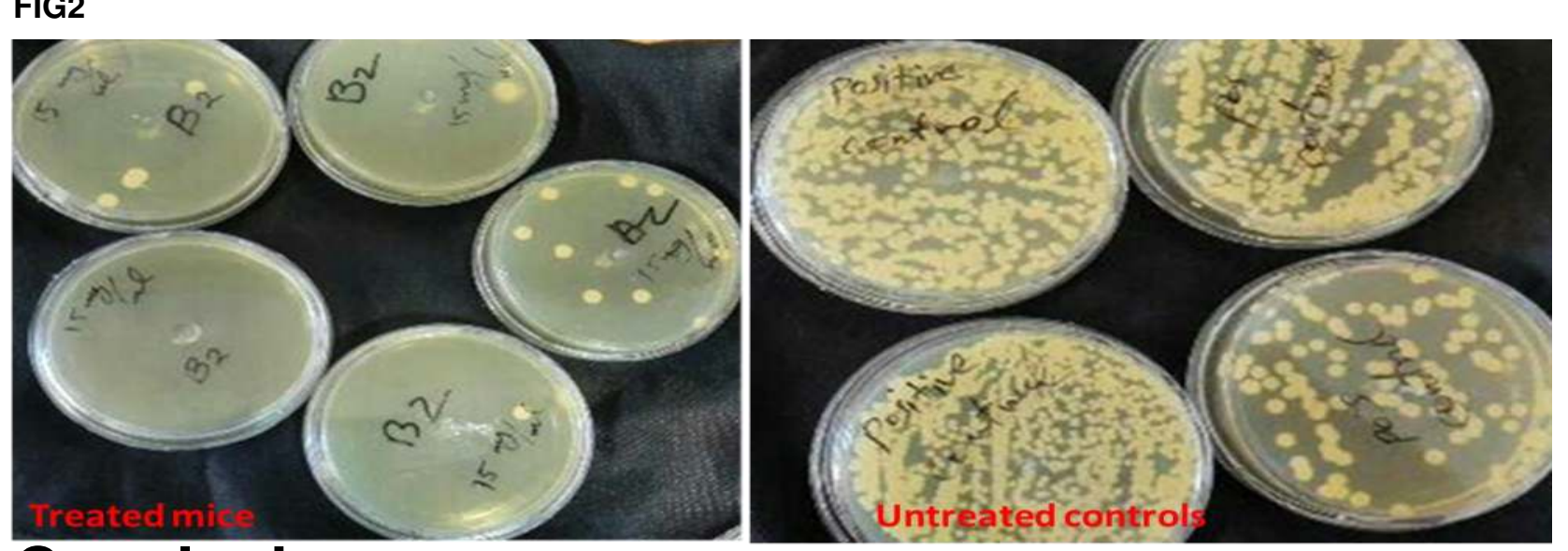

Conclusion: Obtained data provided new insight into the effect of probiotic bacteria on growth, transition, biofilm formation and pathogenicity of Candida and uncover novel probiotic functions. Moreover, the results suggest that probiotics may have the potential to reduce or even inhibit yeast colonization on mucosal surfaces.

Key word: Candida, Pediococcus acidilactici, ALS1, SAP6, EFG1 and HWP1 and quantitative real-time PC 